\title{
The Gaussian CEO Problem for a Scalar Source with Memory: A Necessary Condition
}

\author{
Jie Chen, Feng Jiang and A. Lee Swindlehurst \\ Center for Pervasive Communications and Computing (CPCC) \\ Department of EECS, University of California, Irvine \\ Irvine, CA 92697, USA \\ \{jie.chen, feng.jiang, swindle\}@uci.edu
}

\begin{abstract}
In wireless sensor networks, many monitoring problems can be cast in the form of distributed data collection and centralized data fusion. If the data links from the sensor nodes to the fusion center have limited capacity, there is a tradeoff between estimation precision and transmission rate. This kind of decentralized estimation system is equivalent to the so-called indirect multiterminal source coding problem, and the BergerTung inner bound is the best known achievable rate region boundary. In this paper, we attempt to evaluate the Berger-Tung achievable sum rate for a Gaussian scalar source with arbitrary memory, and we find an analytic necessary condition which the solution to the sum rate problem must satisfy. First, we describe the system model and discuss how the arbitrary memory problem can be tackled by utilizing known results for the vector central estimation officer (CEO) problem. Then we formulate the sum rate calculation as a variational calculus problem with a distortion constraint, and show how to find the necessary condition. Based on our analytic form of the condition, we discuss how to compute the rate-distortion curve and an example is given. We also note that the solution is compatible with previous findings in rate distortion theory, which supports the validity of the necessary condition.
\end{abstract}

\section{INTRODUCTION}

Wireless sensor networks have been the subject of active research for the past decade, and they find many uses in civil, industrial, commercial and military applications. Such networks are often used for distributed sensing, in which geographically distributed sensors make measurements or local estimates and forward them to a fusion center, which further conducts the processing necessary to extract useful information from the reported data. In practice, the local measurements have to be quantized prior to transmission, and there is clearly a tradeoff between the level of quantization (or equivalently the sensor's transmission rate) and the final estimation accuracy. With knowledge of the required estimation accuracy and the statistical characteristics of the source and noise, the fusion center can optimally determine the sensors' individual transmission rates and feed this information back to the sensor nodes in order to efficiently use the available computing and communication resources.

This kind of system is equivalent to the indirect multiterminal source coding problem, which was first studied in [1] and referred to as the central estimation officer (CEO) problem. For memoryless Gaussian scalar sources, the rate region of the $\mathrm{CEO}$ problem has been completely characterized in [2], [3], [4]. As an extension to the scalar problem, the CEO model with a vector source was also considered in [5], [6], [7]. However, except a brief discussion in [8], previous studies all assume the sample sequence generated by the source is memoryless. In this paper, we attempt to evaluate the Berger-Tung achievable sum rate for a Gaussian scalar source with arbitrary memory, and we find an analytic necessary condition which the solution to the sum rate problem must satisfy. First, we describe the system model and discuss how the arbitrary memory problem can be fully established by utilizing known results for the vector CEO problem. Then we formulate the sum rate calculation as a variational calculus problem with a distortion constraint, and show how to find the necessary condition. Based on our analytic form of the condition, we discuss how to compute the rate-distortion curve and an example is given. We also note that the solution is compatible with previous findings in rate distortion theory, which supports the validity of the necessary condition.

The rest of the paper is organized as follows. In Section II, we describe the system model and in Section III we formulate the sum-rate optimization problem for the two-terminal source coding case. Section IV then describes how we can find the necessary condition. An example for a first-order GaussMarkov source is given to show the effectiveness of our approach. We also indicate that the solution is compatible with known results. Section V summarizes the paper and discusses potential future work. The proof of the main theorem used in the paper is deferred to the Appendix.

\section{SySTEM MODEL}

Without loss of generality, we assume there are two sensor nodes in the system. The analysis in this paper can be naturally extended to the case of more than two nodes. A block diagram model is illustrated in Fig. 1.

The fusion center is interested in recovering the real-valued discrete-time source sequence $x(t), t=0, \pm 1, \ldots$, where every $x(t)$ is a Gaussian random variable with zero mean. The source sequence is assumed to be a stationary stochastic process with arbitrary memory; i.e., in contrast to an i.i.d. Gaussian source, the power spectral density (PSD) of $x(t)$, denoted by $\Phi_{x}(\omega)$, is not necessarily constant over frequency. We use $v_{i}(t)$ to indicate the measurement noise (or local estimation error) at sensor node $i$, and we assume it is a 


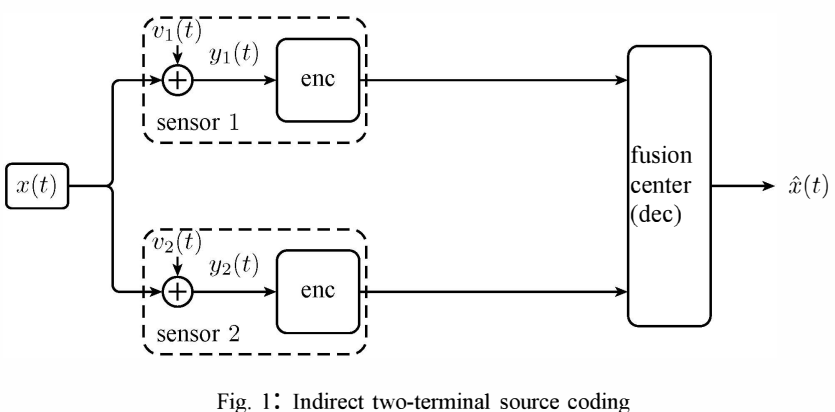

Fig. 1: Indirect two-terminal source coding

real-valued zero-mean Gaussian process and i.i.d. in time. The observation at sensor $i$ is given by $y_{i}(t)=x(t)+v_{i}(t)$, and $\hat{x}(t)$ is the estimate obtained by the fusion center.

We then utilize the technique introduced in [9] to evaluate the Berger-Tung achievable sum rate, $R(D)$, for a given target distortion $D$. Instead of directly calculating the sum rate for a Gaussian source with arbitrary memory, we begin with the evaluation of the achievable sum rate, $R_{n}(D)$, for a Gaussian source word $\mathrm{x}=(x(1), \ldots, x(n))$ and the corresponding reconstructed word $\hat{\mathbf{x}}=(\hat{x}(1), \ldots, \hat{x}(n))$ with the distortion constraint $E\left\{\frac{1}{n} \sum_{t=1}^{n}(x(t)-\hat{x}(t))^{2}\right\} \leq D$. Letting the size of the source word $\mathrm{x}$ go to infinity, we then have $R(D)=$ $\lim _{n \rightarrow \infty} R_{n}(D)$. Similarly the distortion constraint becomes $E\left\{(x(t)-\hat{x}(t))^{2}\right\} \leq D$ because of the stationarity assumption of the source process.

For the purpose of completeness, here we briefly introduce the evaluation of the Berger-Tung sum rate for the problem of limited-size vectors. Let $\mathbf{y}_{1}$ and $\mathbf{y}_{2}$ denote the noise-corrupted observation vectors at the sensor nodes. From the discussion above we know that $\mathrm{y}_{1}$ and $\mathrm{y}_{2}$ are also Gaussian distributed. Based on [4], [5], [6], if there exist auxiliary random vectors $\mathbf{w}_{1}$ and $\mathbf{w}_{2}$ such that $\mathbf{w}_{i} \rightarrow \mathbf{y}_{i} \rightarrow\left(\mathbf{x}, \mathbf{y}_{\{1,2\} \backslash i}, \mathbf{w}_{\{1,2\} \backslash i}\right)$ forms a Markov chain, and if the distortion between $\mathrm{x}$ and $\hat{\mathrm{x}}$ is no greater than $D$, the Berger-Tung achievable rate region is the convex hull of

$$
\begin{aligned}
R\left(\mathbf{w}_{1}, \mathbf{w}_{2}\right)=\left\{\left(R_{1}, R_{2}\right) \mid\right. & R_{1} \geq I\left(\mathbf{y}_{1} ; \mathbf{w}_{1} \mid \mathbf{w}_{2}\right), \\
R_{2} & \geq I\left(\mathbf{y}_{2} ; \mathbf{w}_{2} \mid \mathbf{w}_{1}\right), \\
R_{1}+R_{2} & \left.\geq I\left(\mathbf{y}_{1}, \mathbf{y}_{2} ; \mathbf{w}_{1}, \mathbf{w}_{2}\right)\right\} .
\end{aligned}
$$

It is known in [7] that the Gaussianity of $\mathbf{y}_{i}$ and $\mathbf{w}_{i}$ results in

$$
\begin{aligned}
R_{n}(D) & =\min _{\mathbf{w}_{1}, \mathbf{w}_{2}} \frac{1}{n} I\left(\mathbf{y}_{1}, \mathbf{y}_{2} ; \mathbf{w}_{1}, \mathbf{w}_{2}\right) \\
& =\min _{\mathbf{w}_{1}, \mathbf{w}_{2}} \frac{1}{2 n} \log \frac{\operatorname{det}\left(\mathbf{C}_{\mathbf{y}}\right) \operatorname{det}\left(\mathbf{C}_{\mathbf{w}}\right)}{\operatorname{det}\left(\mathbf{C}_{\mathbf{y w}}\right)},
\end{aligned}
$$

where $\mathbf{C}_{\mathrm{y}}, \mathrm{C}_{\mathrm{w}}$, and $\mathrm{C}_{\mathrm{yw}}$ are the covariance matrices of $\left(\mathbf{y}_{1}^{T} \mathbf{y}_{2}^{T}\right)^{T},\left(\mathbf{w}_{1}^{T} \mathbf{w}_{2}^{T}\right)^{T}$ and $\left(\mathbf{y}_{1}^{T} \mathbf{y}_{2}^{T} \mathbf{w}_{1}^{T} \mathbf{w}_{2}^{T}\right)^{T}$, respectively. It is easy to show that $\mathbf{C}_{\mathbf{y}}, \mathbf{C}_{\mathbf{w}}$, and $\mathbf{C}_{\mathbf{y w}}$ are all block symmetric Toeplitz matrices.

In the next section, we will see how letting the size of the word $\mathrm{x}$ go to infinity can help us obtain a closed-form expression for the mutual information and the distortion, and hence the problem formulation can be fully established.

\section{PROBLEM FORMULATION}

In this section, we establish the full formulation of our problem. In order to calculate the mutual information, we appeal to an extension of the Toeplitz distribution theorem. We also derive the optimal estimator and the corresponding mean squared error (MSE), which are crucial in defining the distortion constraint. Finally we will see the rate evaluation is actually an infinite-dimensional optimization problem, i.e., a variational problem.

\section{A. Mutual Information}

In [9], we can see that the evaluation of the rate distortion function for a point-to-point problem relies on the use of the Toeplitz distribution theorem [10]. Due to the block structure of the mutual information matrices in (3), we need to apply an extension [11] of the Toeplitz distribution theorem to our problem. To facilitate an understanding of our results, we recapitulate the extension here. Let $\mathbf{T}_{c n \times c n}$ denote a $c \times c$ block matrix with $n \times n$ Toeplitz submatrices as its blocks, i.e.,

$$
\mathbf{T}_{c n \times c n}=\left[\begin{array}{ccc}
T_{1,1} & \cdots & T_{1, c} \\
\vdots & \ddots & \vdots \\
T_{c, 1} & \cdots & T_{c, c}
\end{array}\right]
$$

and

$$
T_{i, j}=\left[\begin{array}{cccc}
T_{i, j}(1) & \ldots & \ldots & T_{i, j}(-n) \\
T_{i, j}(2) & T_{i, j}(1) & \ldots & T_{i, j}(-n+1) \\
\vdots & \ddots & \ddots & \vdots \\
T_{i, j}(n) & \ldots & T_{i, j}(2) & T_{i, j}(1)
\end{array}\right] .
$$

If $T_{i, j}$ is Hermitian, Theorem 3 of [11] says

$\lim _{n \rightarrow \infty} \frac{1}{n} \sum_{k=1}^{c n} F\left(\lambda_{k}\left(\mathbf{T}_{c n \times c n}\right)\right)=\frac{1}{2 \pi} \int_{-\pi}^{\pi} \sum_{u=1}^{c} F\left(\lambda_{u}(\boldsymbol{\Theta}(\omega))\right) d \omega$,

where $F$ is an arbitrary function, $\lambda_{k}$ and $\lambda_{u}$ are respectively the eigenvalues of $\mathbf{T}_{c n \times c n}$ and $\boldsymbol{\Theta}(\omega)$, and

$$
\begin{aligned}
& \boldsymbol{\Theta}(\omega)= \\
& {\left[\begin{array}{ccc}
\sum_{k=-\infty}^{\infty} T_{1,1}(k) e^{-j k \omega} & \ldots & \sum_{k=-\infty}^{\infty} T_{1, c}(k) e^{-j k \omega} \\
\vdots & \ddots & \vdots \\
\sum_{k=-\infty}^{\infty} T_{c, 1}(k) e^{-j k \omega} & \ldots & \sum_{k=-\infty}^{\infty} T_{c, c}(k) e^{-j k \omega}
\end{array}\right] .}
\end{aligned}
$$

Assign the $\log$ function to $F$ and let the size of the matrices in (3) goes to infinity. After applying (4) to (3), we get the rate expression for the sources-with-memory problem:

$$
\begin{aligned}
& I\left(y_{1}(t), y_{2}(t) ; w_{1}(t), w_{2}(t)\right)= \\
& \frac{1}{4 \pi} \int_{-\pi}^{\pi} \operatorname{det}\left[\begin{array}{ccc}
\Phi_{y_{1}} & \Phi_{y_{1} y_{2}} \\
\Phi_{y_{2} y_{1}} & \Phi_{y_{2}}
\end{array}\right] \operatorname{det}\left[\begin{array}{ccc}
\Phi_{w_{1}} & \Phi_{w_{1} w_{2}} \\
\Phi_{w_{2} w_{1}} & \Phi_{w_{2}}
\end{array}\right]
\end{aligned}
$$




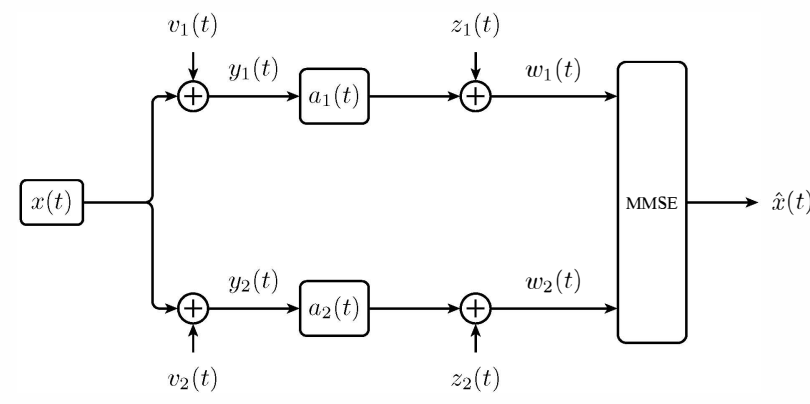

Fig. 2: Forward test channels

where $\Phi_{y_{i}}, \Phi_{y_{i} y_{j}}, \Phi_{w_{i}}, \Phi_{w_{i} w_{j}}, \Phi_{y_{i} w_{j}}, \Phi_{w_{i} y_{j}}$ are the auto- and cross-PSD functions of the corresponding stochastic processes. Note that in general they are all non-constant functions of $\omega$. To make the expression more compact and with a slight abuse of notation, we drop $\omega$ and write $\Phi$ for $\Phi(\omega)$ when it does not cause confusion.

To calculate the mutual information expression of (5), we need to use the concept of the so-called forward test channel [9]. Assuming the outputs of the dequantizers at the fusion center, $w_{i}(t), i=1,2$, are also Gaussian distributed, we can write the whole system in the forward test channel form

$$
\begin{aligned}
& w_{1}(t)=a_{1}(t) * y_{1}(t)+z_{1}(t) \\
& w_{2}(t)=a_{2}(t) * y_{2}(t)+z_{2}(t)
\end{aligned}
$$

where $*$ represents convolution, $a_{i}(t), i=1,2$ are variable real functions to be determined, and $z_{i}(t), i=1,2$ represent quantization noise, which is i.i.d. in time and independent from $y_{i}(t)$. The forward test channels are depicted in Fig. 2. Unlike problems with memoryless sources, convolution is required here instead of simple multiplication. be

Given (6), the auto- and cross-PSD functions are found to

$$
\left\{\begin{array}{ll}
\Phi_{y_{i}}(\omega)=\Phi_{x}(\omega)+\Phi_{v_{i}}(\omega) & \\
\Phi_{w_{i}}(\omega)=\left|A_{i}(\omega)\right|^{2} \Phi_{y_{i}}(\omega)+\Phi_{z_{i}}(\omega) & \\
\Phi_{w_{i} y_{i}}(\omega)=A_{i}(\omega) \Phi_{y_{i}}(\omega) & \\
\Phi_{y_{i} w_{i}}(\omega)=A_{i}^{*}(\omega) \Phi_{y_{i}}(\omega) & \\
\Phi_{x y_{i}}(\omega)=\Phi_{x}(\omega) & \\
\Phi_{x w_{i}}(\omega)=A_{i}^{*}(\omega) \Phi_{x}(\omega) & i \neq j \\
\Phi_{w_{i} y_{j}}(\omega)=A_{i}(\omega) \Phi_{x}(\omega) & i \neq j \\
\Phi_{y_{i} w_{j}}(\omega)=A_{j}^{*}(\omega) \Phi_{x}(\omega) & i \neq j \\
\Phi_{y_{i} y_{j}}(\omega)=\Phi_{x}(\omega) & i \neq j \\
\Phi_{w_{i} w_{j}}(\omega)=A_{i}(\omega) A_{j}^{*}(\omega) \Phi_{x}(\omega) &
\end{array},\right.
$$

where $i$ and $j$ take the values of 1 or 2 .

Plugging (7) into (5) and after a long series of mathematical manipulations, we can simplify (5) to the following expression

$$
\begin{aligned}
& I\left(y_{1}(t), y_{2}(t) ; w_{1}(t), w_{2}(t)\right)= \\
& \frac{1}{4 \pi} \int_{-\pi}^{\pi} \log \frac{\left(\Phi_{y_{1}}+\Phi_{m_{1}}^{-1}\right)\left(\Phi_{y_{2}}+\Phi_{m_{2}}^{-1}\right)-\Phi_{x}^{2}}{\Phi_{m_{1}}^{-1} \Phi_{m_{2}}^{-1}} d \omega,
\end{aligned}
$$

where $\Phi_{m_{i}}(\omega)=\left(\left|A_{i}(\omega)\right|^{-2} \Phi_{z_{i}}(\omega)\right)^{-1}, \Phi_{z_{i}}(\omega)$ is the PSD of $z_{i}(t)$, and $A_{i}(\omega)$ is the Fourier transform of $a_{i}(t)$.

\section{B. MSE and Optimal Estimator}

In order to obtain a solution to the minimum sum rate problem, we also need an explicit expression for the distortion. In our case, we choose MSE, the most commonly used distortion metric, as our distortion objective. For memoryless sources, minimizing the MSE between $x(t)$ and $\hat{x}(t)$ can be achieved by means of a simple MMSE estimator. Our problem involving sources with arbitrary memory is much more complicated. Here we want to estimate an entire stochastic process using two infinite-length sequences as the input. The estimator will have the form $\hat{x}(t)=\left[h_{1}(t) h_{2}(t)\right] *\left[w_{1}(t) w_{2}(t)\right]^{T}$, where $h_{i}(t)$ for $i=1,2$ are optimal infinite-length filters. For known $h_{1}(t)$ and $h_{2}(t)$, the MSE is given by

$$
\begin{aligned}
& E\left\{(x(t)-\hat{x}(t))^{2}\right\} \\
& =\frac{1}{2 \pi} \int_{-\pi}^{\pi}\left(\Phi_{x}(\omega)-\sum_{i=1}^{2} \Phi_{x w_{i}}(\omega) H_{i}^{*}(\omega)\right) d \omega,
\end{aligned}
$$

where $H_{i}(\omega)$ is the Fourier transform of $h_{i}(t), \Phi_{x w_{i}}(\omega)$ is the cross-PSD for $x(t)$ and $w_{i}(t)$, and $H_{i}^{*}(\omega)$ is the conjugate of $H_{i}(\omega)$.

The optimal estimator can be obtained by solving the frequency domain Wiener-Hopf equations [12]:

$$
\left[\begin{array}{c}
H_{1}(\omega) \\
H_{2}(\omega)
\end{array}\right]^{T}\left[\begin{array}{cc}
\Phi_{w_{1}}(\omega) & \Phi_{w_{1} w_{2}}(\omega) \\
\Phi_{w_{2} w_{1}}(\omega) & \Phi_{w_{2}}(\omega)
\end{array}\right]=\left[\begin{array}{c}
\Phi_{x w_{1}}(\omega) \\
\Phi_{x w_{2}}(\omega)
\end{array}\right]^{T} .
$$

The solution to (10) is

$$
\begin{aligned}
{\left[\begin{array}{c}
H_{1}(\omega) \\
H_{2}(\omega)
\end{array}\right]^{T}=} & {\left[\left|A_{1}\right|^{2}\left|A_{2}\right|^{2}\left(\Phi_{x} \Phi_{v_{1}}+\Phi_{x} \Phi_{v_{2}}+\Phi_{v_{1}} \Phi_{v_{2}}\right)\right.} \\
& \left.+\left|A_{1}\right|^{2} \Phi_{y_{1}} \Phi_{z_{2}}+\left|A_{2}\right|^{2} \Phi_{y_{2}} \Phi_{z_{1}}+\Phi_{z_{1}} \Phi_{z_{2}}\right]^{-1} \\
& \times\left[\begin{array}{c}
A_{1}^{*} \Phi_{x}\left(\left|A_{2}\right|^{2} \Phi_{v_{2}}+\Phi_{z_{2}}\right) \\
A_{2}^{*} \Phi_{x}\left(\left|A_{1}\right|^{2} \Phi_{v_{1}}+\Phi_{z_{1}}\right)
\end{array}\right]^{T} .
\end{aligned}
$$

We reiterate that $A_{i}$ and all $\Phi$ 's are functions of $\omega$, which has simply been dropped to save space. Plugging the resulting $H_{i}(\omega)$ into (9), after some simplifications we obtain

$$
\begin{aligned}
& E\left\{(x(t)-\hat{x}(t))^{2}\right\} \\
& =\frac{1}{2 \pi} \int_{-\pi}^{\pi} \frac{1}{\frac{1}{\Phi_{x}(\omega)}+\frac{1}{\Phi_{v_{1}}(\omega)+\frac{1}{\Phi_{m_{1}}(\omega)}}+\frac{1}{\Phi_{v_{2}}(\omega)+\frac{1}{\Phi_{m_{2}}(\omega)}}} d \omega .
\end{aligned}
$$

\section{Full Formulation}

By assuming that the rate is minimized when the distortion target is achieved with equality, the rate evaluation problem turns out to have the form shown below.

$$
\begin{aligned}
& \min _{\Phi_{m_{1}}, \Phi_{m_{2}}} \frac{1}{4 \pi} \int_{-\pi}^{\pi} \log \frac{\left(\Phi_{y_{1}}+\Phi_{m_{1}}^{-1}\right)\left(\Phi_{y_{2}}+\Phi_{m_{2}}^{-1}\right)-\Phi_{x}^{2}}{\Phi_{m_{1}}^{-1} \Phi_{m_{2}}^{-1}} d \omega \\
& \text { s.t. } \frac{1}{2 \pi} \int_{-\pi}^{\pi} \frac{1}{\frac{1}{\Phi_{x}}+\frac{1}{\Phi_{v_{1}}+\frac{1}{\Phi_{m_{1}}}}+\frac{1}{\Phi_{v_{2}}+\frac{1}{\Phi_{m_{2}}}}} d \omega=D \\
& \Phi_{m_{1}} \geq 0 \\
& \Phi_{m_{2}} \geq 0 .
\end{aligned}
$$




$$
\begin{gathered}
\Phi_{m_{1}}=\max \left(0, \frac{-\left(\Phi_{v_{1}}^{2}\left(\Phi_{x}+\Phi_{v_{2}}\right)^{2}+\frac{1}{2} \Phi_{x} \Phi_{v_{1}} \Phi_{v_{2}}\left(-\lambda \Phi_{x} \Phi_{v_{2}}+\Phi_{x}+\Phi_{v_{2}}\right)-\frac{1}{2} \Phi_{x}^{2} \Phi_{v_{2}}^{2}-\frac{1}{2} \Phi_{x} \Phi_{v_{2}} \Xi\right)}{\Phi_{v_{1}}\left(\Phi_{x} \Phi_{v_{1}}+\Phi_{x} \Phi_{v_{2}}+\Phi_{v_{1}} \Phi_{v_{2}}\right)^{2}}\right) \\
\Phi_{m_{2}}=\max \left(0, \frac{-\left(\Phi_{v_{2}}^{2}\left(\Phi_{x}+\Phi_{v_{1}}\right)^{2}+\frac{1}{2} \Phi_{x} \Phi_{v_{1}} \Phi_{v_{2}}\left(-\lambda \Phi_{x} \Phi_{v_{1}}+\Phi_{x}+\Phi_{v_{1}}\right)-\frac{1}{2} \Phi_{x}^{2} \Phi_{v_{1}}^{2}-\frac{1}{2} \Phi_{x} \Phi_{v_{1}} \Xi\right)}{\Phi_{v_{2}}\left(\Phi_{x} \Phi_{v_{1}}+\Phi_{x} \Phi_{v_{2}}+\Phi_{v_{1}} \Phi_{v_{2}}\right)^{2}}\right) \\
\Xi= \pm\left(\lambda^{2} \Phi_{x}^{2} \Phi_{v_{1}}^{2} \Phi_{v_{2}}^{2}+6 \lambda \Phi_{x}^{2} \Phi_{v_{1}}^{2} \Phi_{v_{2}}+6 \lambda \Phi_{x}^{2} \Phi_{v_{1}} \Phi_{v_{2}}^{2}+6 \lambda \Phi_{x} \Phi_{v_{1}}^{2} \Phi_{v_{2}}^{2}\right. \\
\left.\quad+\Phi_{x}^{2} \Phi_{v_{1}}^{2}+2 \Phi_{x}^{2} \Phi_{v_{1}} \Phi_{v_{2}}+\Phi_{x}^{2} \Phi_{v_{2}}^{2}+2 \Phi_{x} \Phi_{v_{1}}^{2} \Phi_{v_{2}}+2 \Phi_{x} \Phi_{v_{1}} \Phi_{v_{2}}^{2}+\Phi_{v_{1}}^{2} \Phi_{v_{2}}^{2}\right)^{\frac{1}{2}}
\end{gathered}
$$

This is a functional optimization problem and can be tackled through the calculus of variations.

\section{NeCESSARY CONDITION}

In this section we show an analytic necessary condition which the solution to the sum rate problem must satisfy. We refer to it as a necessary condition because if we can find the optimal sum rate then it must agree with our closed-form condition, but theoretically it is possible that our condition could also lead to a non-optimal sum rate.

As we have seen in Section III, the optimization in its final form is a constrained variational problem. In fact it can be regarded as an isoperimetric problem with additional inequality constraints. The following theorem shows that the solution consists of a zero part and a non-zero part, and the non-zero part can be determined by solving a set of Euler equations. As known from many texts on the calculus of variations, solving Euler equations results in a necessary condition.

Theorem 1 (Isoperimetric problem with additional inequality constraints). Let $u_{i}$ and $\psi_{i}, i=1, \ldots, n$ be functions of $\omega$. For the following functional optimization problem,

$$
\begin{gathered}
\min _{u_{1}, \ldots, u_{n}} J\left(u_{1}, \ldots, u_{n}\right)=\int_{a}^{b} f\left(\omega, u_{1}, \ldots, u_{n}, u_{1}^{\prime}, \ldots, u_{n}^{\prime}\right) d \omega \\
\text { s.t. } \int_{a}^{b} g\left(\omega, u_{1}, \ldots, u_{n}, u_{1}^{\prime}, \ldots, u_{n}^{\prime}\right) d \omega=D \\
\quad u_{i} \geq \psi_{i}, i=1, \ldots, n,
\end{gathered}
$$

the solution $u_{i}$ should consist of two parts: $\psi_{i}$ and a function no less than $\psi_{i}$. The latter can be obtained through solving the Euler equations

$$
f_{u_{i}}-\frac{d}{d \omega} f_{u_{i}^{\prime}}+\lambda\left(g_{u_{i}}-\frac{d}{d \omega} g_{u_{i}^{\prime}}\right)=0, i=1 \ldots, n .
$$

Proof: See the appendix for the proof.

In our problem, $n=2, u_{i}=\Phi_{m_{i}}$ and $\psi_{i}=0$, and we can directly apply Theorem 1. Due to the fact that there are no derivatives of $\Phi_{i}(\omega)$ in the integrand of the objective function, the Euler equations turn out to be merely algebraic rather than differential equations. After eliminating the infeasible solutions to these equations, we end up with the solution shown in (21)-(23). Here the Lagrange multiplier $\lambda$ should be carefully picked so that (14) is fulfilled.

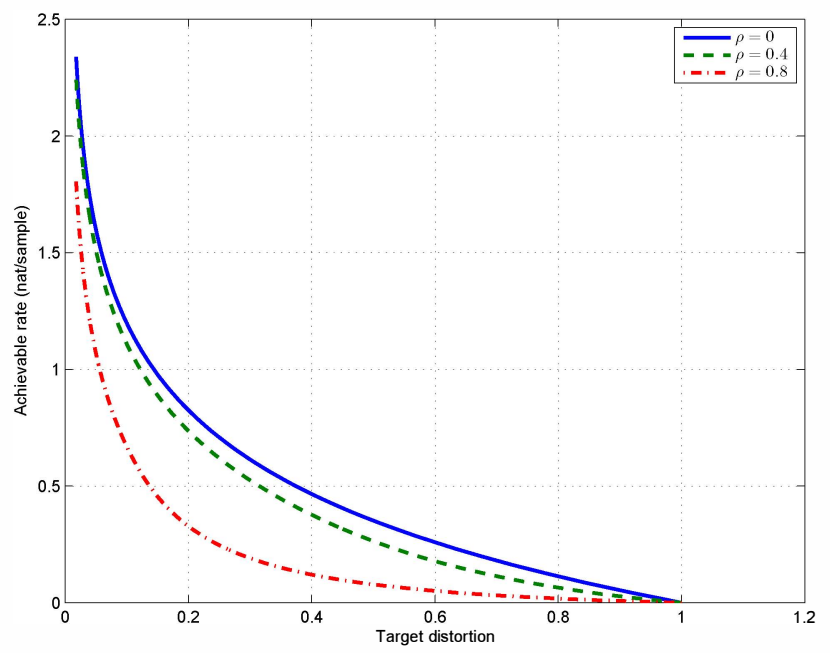

Fig. 3: Achievable sum rate versus target distortion

Remark 1 (Evaluation of the achievable sum rate function). We now explain how to use our solution to calculate the achievable sum rate as a function of $D$. For a given $D$, normally we need to plug (21) and (22) into (14) and then solve it to get the value of the Lagrange multiplier $\lambda$, which will be further plugged into (8) to completely determine the achievable rate $R$. As an example, we show three rate curves for a first-order Gauss-Markov source in Fig. 3. The power of the source process is 1 . The noise powers at both sensors are 0.01 . The PSD of this source process is $\frac{1-\rho^{2}}{1+\rho^{2}-2 \rho \cos \omega}$, where $\rho$ is the correlation coefficient. Three rate curves, corresponding to $\rho=0,0.4$, and 0.8 , are shown. As expected, the rate requirement decreases as the correlation increases.

Due to the existence of the integration and minimum operators, sometimes it is difficult to use the above method to evaluate the sum rate. Another approach, which is similar to the one explained in [9], is to first pick a value for $\lambda$, then plug it into (21), (22), (13) and (14) to get the corresponding sum rate $R$ and distortion $D$. The entire achievable rate-distortion curve can be found in this fashion.

Remark 2 (Compatibility with known results). We have obtained the solution to a very general problem which should cover cases like the problem of rate evaluation for sources without memory. For this case, it is easy to show via a simple 
notation change that the functional optimization problem of (13)-(16) is exactly the same as the multiterminal Berger-Tung achievable rate bound for i.i.d. Gaussian sources described in [4]. Numerical experiments also show that they generate the same rate-distortion curve.

\section{CONCLUSiOn}

In this paper, we have attempted to evaluate the Berger-Tung achievable sum rate for a Gaussian scalar source with arbitrary memory, and we have found an analytic necessary condition which the solution to the sum rate problem must satisfy. We also discussed various aspects of the solution, including how to evaluate the rate-distortion curve and compatibility with previous results. Possible future work includes evaluating the rate function for specific source processes such as high-order Gauss-Markov processes. Most importantly, we want to find a sufficient condition which the sum rate solution must satisfy, so that we can determine the source conditions under which the sum rate can be calculated using the method explained in this paper.

\section{APPENDIX}

Here we provide a proof of Theorem 1, which to our knowledge is not found in texts on the calculus of variations.

Proof: Since $u_{i} \geq \psi_{i}, i=1, \ldots, n$, there exist real functions $z_{i}, i=1, \ldots, n$ such that we can write $u_{i}=\psi_{i}+z_{i}^{2}$ for all $i$. Substituting $\psi_{i}+z_{i}^{2}$ for $u_{i}$ into the functional optimization problem in Theorem 1, we end up with

$$
\begin{aligned}
\min _{z_{1}, \ldots, z_{n}} & \int_{a}^{b} F\left(\omega, z_{1}, \ldots, z_{n}, z_{1}^{\prime}, \ldots, z_{n}^{\prime}\right) d \omega \\
\text { s.t. } & \int_{a}^{b} F\left(\omega, z_{1}, \ldots, z_{n}, z_{1}^{\prime}, \ldots, z_{n}^{\prime}\right) d \omega=D,
\end{aligned}
$$

where

$$
\begin{aligned}
& F\left(\omega, z_{1}, \ldots, z_{n}, z_{1}^{\prime}, \ldots, z_{n}^{\prime}\right)= \\
& \left.\quad f\left(\omega, u_{1}, \ldots, u_{n}, u_{1}^{\prime}, \ldots, u_{n}^{\prime}\right)\right|_{u_{i}=\psi_{i}+z_{i}^{2}}
\end{aligned}
$$

and

$$
\begin{aligned}
& G\left(\omega, z_{1}, \ldots, z_{n}, z_{1}^{\prime}, \ldots, z_{n}^{\prime}\right)= \\
& \left.\quad g\left(\omega, u_{1}, \ldots, u_{n}, u_{1}^{\prime}, \ldots, u_{n}^{\prime}\right)\right|_{u_{i}=\psi_{i}+z_{i}^{2}} .
\end{aligned}
$$

It is clear that solving this problem for $z_{i}$ is equivalent to finding the optimal $u_{i}$.

The recast problem is an isoperimetric problem, which can be solved by using the method of Lagrange multipliers. Letting $\lambda$ denote the multiplier, we have the Lagrangian as

$$
\begin{aligned}
& \int_{a}^{b} F\left(\omega, z_{1}, \ldots, z_{n}, z_{1}^{\prime}, \ldots, z_{n}^{\prime}\right) d \omega \\
& +\lambda \int_{a}^{b} G\left(\omega, z_{1}, \ldots, z_{n}, z_{1}^{\prime}, \ldots, z_{n}^{\prime}\right) d \omega .
\end{aligned}
$$

Calculate the derivative of the Lagrangian with respect to $z_{i}$ for all $i$ and set them equal to zero:

$$
F_{z_{i}}-\frac{d}{d \omega} F_{z_{i}^{\prime}}+\lambda\left(G_{z_{i}}-\frac{d}{d \omega} G_{z_{i}^{\prime}}\right)=0 .
$$

Here we follow the notation convention in [13] and the subscripts signify the partial derivatives with respect to the corresponding functions.

Since

$$
F_{z_{i}}=2 z_{i} f_{u_{i}}+2 z_{i}^{\prime} f_{u_{i}^{\prime}}
$$

and

$$
F_{z_{i}^{\prime}}=2 z_{i} f_{u_{i}^{\prime}}
$$

equation (29) becomes

$$
z_{i}\left(f_{u_{i}}-\frac{d}{d \omega} f_{u_{i}^{\prime}}+\lambda\left(g_{u_{i}}-\frac{d}{d \omega} g_{u_{i}^{\prime}}\right)\right)=0,
$$

which means either $z_{i}=0$ or $f_{u_{i}}-\frac{d}{d \omega} f_{u_{i}^{\prime}}+\lambda\left(g_{u_{i}}-\frac{d}{d \omega} g_{u_{i}^{\prime}}\right)=$ 0 . If $z_{i}=0, u_{i}$ should be equal to $\psi_{i}$. Otherwise it should be the solution to

$$
f_{u_{i}}-\frac{d}{d \omega} f_{u_{i}^{\prime}}+\lambda\left(g_{u_{i}}-\frac{d}{d \omega} g_{u_{i}^{\prime}}\right)=0, i=1, \ldots, n,
$$

which are exactly the Euler equations. This concludes the proof.

\section{REFERENCES}

[1] T. Berger, Zhen Zhang, and H. Viswanathan, "The CEO problem," IEEE Trans. Inf. Theory, vol. 42, no. 3, pp. 887-902, 1996

[2] Y. Oohama, "The rate-distortion function for the quadratic Gaussian CEO problem," IEEE Trans. Inf. Theory, vol. 44, no. 3, pp. 1057-1070, 1998.

[3] V. Prabhakaran, D. Tse, and K. Ramachandran, "Rate region of the quadratic Gaussian CEO problem," in Proc. Int. Symp. Information Theory ISIT 2004, 2004.

[4] Jun Chen, Xin Zhang, T. Berger, and S. B. Wicker, "An upper bound on the sum-rate distortion function and its corresponding rate allocation schemes for the CEO problem," IEEE J. Sel. Areas Commun., vol. 22, no. 6, pp. 977-987, 2004

[5] S. Tavildar and P. Viswanath, "On the sum-rate of the vector Gaussian CEO problem," in Proc. Conf Signals, Systems and Computers Record of the Thirty-Ninth Asilomar Conf, 2005, pp. 3-7.

[6] Jin-Jun Xiao and Zhi-Quan Luo, "Optimal rate allocation for the vector Gaussian CEO problem," in Proc. 1st IEEE Int Computational Advances in Multi-Sensor Adaptive Processing Workshop, 2005, pp. 56-59.

[7] Jie Chen and A. Lee Swindlehurst, "On the achievable sum rate of multiterminal source coding for a correlated Gaussian vector source," in Proc. IEEE International Conf. on Acoustics Speech and Signal Processing (ICASSP), March 2012

[8] S. Tung, Multiterminal Rate-Distortion Theory, Ph.D. thesis, Cornell University, School of Elec. Eng., Ithaca, NY, 1978.

[9] T. Berger, Rate Distortion Theory: A Mathematical Basis for Data Compression, Prentice-Hall, 1971.

[10] U. Grenander and G. Szegö, Toeplitz Forms and Their Applications, Chelsea Publishing Company, 1964

[11] H. Gazzah, P. A. Regalia, and J.-P. Delmas, "Asymptotic eigenvalue distribution of block Toeplitz matrices and application to blind SIMO channel identification," IEEE Trans. Inf. Theory, vol. 47, no. 3, pp. 1243-1251, 2001.

[12] T. Kailath, A.H. Sayed, and B. Hassibi, Linear Estimation, Prentice Hall, 2000.

[13] I.M. Gelfand and S.V. Fomin, Calculus of Variations, Dover Books on Mathematics. Dover Publications, 2000 